\title{
All Aspects of Antioxidant Properties of Kombucha Drink
}

\author{
Ramona Massoud ${ }^{1(\mathbb{D})}$, Reyhaneh Jafari-Dastjerdeh ${ }^{2}{ }^{\mathbb{D}}$, Nafisesadat Naghavi ${ }^{3}{ }^{\mathbb{D}}$, Kianoush Khosravi- \\ Darani 4 ,*(D)
}

1 Department of Food Science and Technology, Iran Standard Organization Tehran, Iran; rm8059@yahoo.com (R.M.);

2 Department of Microbiology, Falavarjan Branch, Islamic Azad University, Falavarjan, Isfahan, Iran; Reyhanehjafari24@yahoo.com (R.J.D.);

3 Department of Microbiology, Falavarjan Branch, Islamic Azad University, Falavarjan, Isfahan, Iran; Nafiseh_naghavy@yahoo.com (N.S.N.);

4 Research Department of Food Technology, National Nutrition and Food Technology Research Institute, Faculty of Nutrition Sciences and Food Technology, Shahid Beheshti University of Medical Sciences, Tehran, Iran; kiankh@yahoo.com (K.K.D.);

* Correspondence: kiankh@yahoo.com;

Scopus Author ID 23969408200

Received: 7.02.2021; Revised: 10.05.2021; Accepted: 14.05.2021; Published: 13.08.2021

Abstract: Nowadays people chose their food and drinks carefully as they are aware of the role of their diet in staying healthy. Human beings are looking for safe, healthy food to get the essential nutrients and have health effects simultaneously. Kombucha, a unique drink, is claimed to have various beneficial effects, and the antioxidant activity is one of the most important ones. Kombucha is mainly based on black and green tea and sugar, but meanwhile, it has been produced by many additional raw materials such as fruit peels, rice, wheat, barley, and honey to add nutritional value and consumer acceptance. This review paper describes the fermentation, biochemistry, and microbiology of kombucha, and also the chemical composition of kombucha was explained along with the main factors affecting the properties of kombucha. This study reviews all aspects of kombucha's antioxidant properties and mentioned the main factors affecting its antioxidant activity.

Keywords: kombucha; tea; microbial starter; antioxidant; fermentation.

(C) 2021 by the authors. This article is an open-access article distributed under the terms and conditions of the Creative Commons Attribution (CC BY) license (https://creativecommons.org/licenses/by/4.0/).

\section{Introduction}

Recently the herbal drink consumption has increased all over the world due to having beneficial health impacts. These beverages are prepared from plant material, placing a small in freshly boiled water. Among all, black and green tea are the most popular ones [1]. Tea is cultivated in many parts of the world, in nearly 30 countries, and is popular after water [2]. Many studies have been proved the antioxidant effects of tea. Polyphenols and catechins are the most important antioxidants in tea that belong to the flavonols group [3,4]. Polyphenols are notable for their high antioxidant traits [2,5]. Polyphenols make up nearly $30 \%$ of the tea leave's dry weight [6]. Tea leaves are a suitable substrate for fermenting kombucha $[7,8]$. Kombucha is a traditional fermented beverage with a history from the East, and now it is quite well known globally [9-10]. 


\section{Kombucha Tea}

\subsection{History of kombucha.}

More than 2,500 years ago, Manchuria's people invented a fermented beverage with a sweet and sour taste that soon became popular throughout China. By traveling this beverage to Japan, they called it kombucha, which comes from Kombo that means algae, and cha which means tea [11]. By the expansion of trade routes, kombucha tea found its way to migrate around the world, and it soon spread all over the world [11]. Kombucha fermented tea belongs to the family of fermented or processed foods, such as yogurt, vinegar, and cheese, consumed for their benefits for centuries. In general, fermented food results from microorganism's activities that make changes in the taste, nutritional value, and digestibility of the primary food [12]. Kombucha results from the symbiosis of a group of eukaryotic such as yeasts and prokaryotes such as bacteria, which are grown in a suitable environment such as sweet tea. These microorganisms would produce various useful compounds while growing and multiplying in the sweet tea culture medium [13].

\subsection{Preparation method of kombucha tea.}

Black and green tea sweetened with sucrose is the most common substrates for this fermented drink. The amount and size of the Symbiotic Community of Bacteria and Yeast (SCOBY) may also vary in the formulation. A standard preparation method for kombucha tea was described in Fig 1 [14]. In this method, 50 to $70 \mathrm{~g}$ of sucrose is dissolved in 1 liter of boiling water, then the black or green tea leaves are added, and after 5 minutes, the leaves are removed by using a filter. In the next step, the SCOBY culture of kombucha and a cellulose layer is added to the solution. It is covered with a clean cloth and remained for 8-14 days in a dark place for a complete fermentation [14].

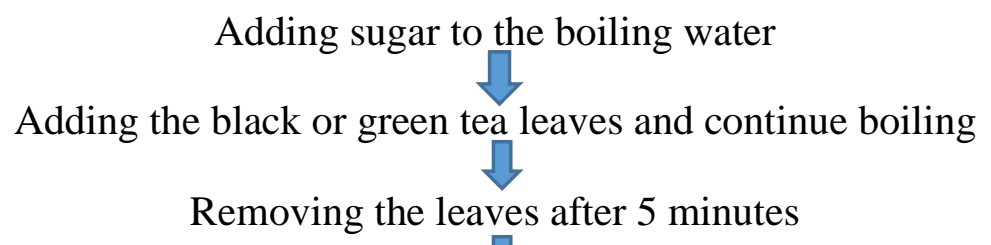

Adding the SCOBY culture of kombucha and a piece of cellulose layer

Covering with a clean cloth to start the fermentation level for 8-14 days

Figure 1. A schematic process for the production of kombucha drinks.

\subsection{Fermentation and biochemistry of kombucha.}

The traditional substrate for kombucha's fermentation is black or green tea extract with 5 to $8 \%$ sucrose. The sweetened tea with sugar is usually used to produce kombucha. Sucrose is the main carbon source in this process [15]. The sucrose in tea is hydrolyzed to fructose and glucose by the invertase produced by the yeasts in kombucha. First, glucose is converted to ethanol and carbon dioxide by yeasts [16]. Then ethanol is converted to acetic acid by acetobacteria. This is the main fermentation route of kombucha; ethanol, gluconic acid, and acetic acid are the main kombucha tea products [17]. The important metabolic activity of kombucha tea is shown schematically in Fig 2 [18]. 


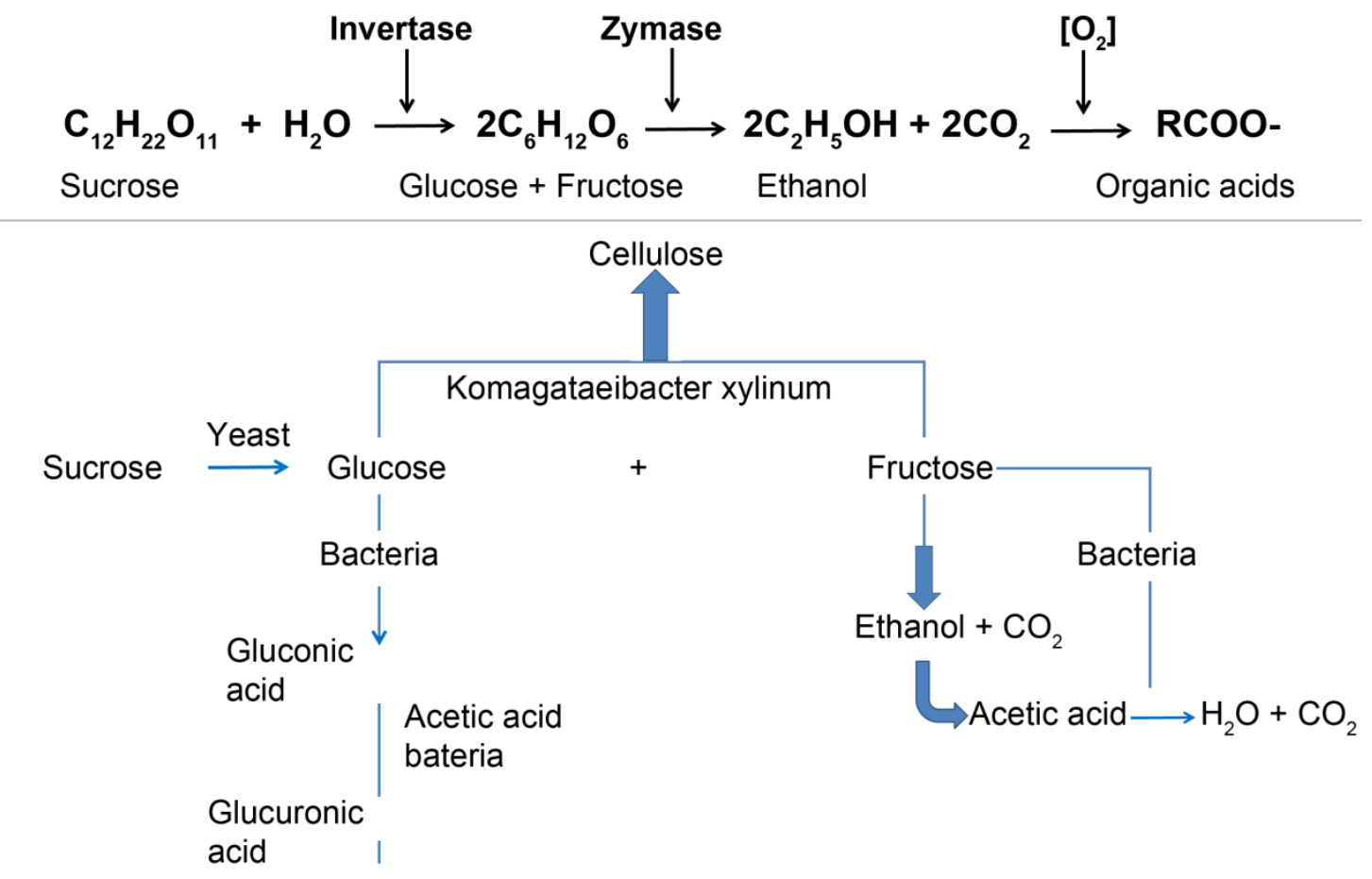

Figure 2. The main fermentation route of kombucha.

\subsection{Microbiology of kombucha.}

Kombucha is a symbiotic mass of bacteria (often acetobacter) and osmophilic yeasts and is a flat, smooth and dense plate. Some new layers are created on the first plate during the fermentation process that would be separated from the previous layers. First, the fungus forms a layer on the tea's surface that gradually thickens later [19-22]. Bacteria such as Acetobacter xylinum, Acetobacter xylinoide, Acetobacter aceti, Acetobacter pasteurianus, Bacterium gluconicum, and yeasts like Zygosaccharomyces bailii, Schizo saccharomyces pombe, Saccharomycodes ludwigii, Berttanomyces bruxellensis, Candida kefyr, and Pichia sp. have been isolated from this biomass [23-27].

\subsection{Chemical composition of kombucha.}

There are many chemical compounds in kombucha drink such as acetic, folic, lactic, carbonic gluconic, glucuronic, oxalic, malic and citric acids, vitamin $\mathrm{C}, \mathrm{B}_{1}, \mathrm{~B}_{3}, \mathrm{~B}_{12}$ as well as main tea components such as catechins, tiaflavin, and flavonols, and some enzymes including invertase, amylase and other oxidative enzymes [28]. Usnic acid is one of the natural antibiotics in kombucha, which has antibacterial and antiviral properties. It is effective against grampositive bacteria such as Enterococcus faecalis and Staphylococcus aureus and [29, 30].

\subsection{Antioxidant activity of kombucha.}

Polyphenols make up nearly $30 \%$ of the fresh tea leaves dry weight. The famous polyphenols in tea are epigallocatechin, epicatechin. Kombucha's free radical scavenging activity is higher when brewed with green tea [6]. The breakage of complex molecules in kombucha tea occurs when the enzymes are released by bacteria and yeast and affect the phenolic compounds. The total phenolic compounds in tea are increased. Therefore, the total phenolic content improves after fermentation [2]. However, an excessive increase in 
fermentation time is not appropriate because the accumulation of organic acids can adversely affect kombucha tea [2]. This fungus has strong antioxidant properties, reducing the degree of fat oxidation and preventing DNA fragmentation [10].

Like black tea, kombucha contains polyphenols and other compounds with antioxidant power, but it has more beneficial effects than regular black tea due to fermentation. Food experts have found that the kombucha's antioxidant activity is more than vitamin $\mathrm{C}$ and $\mathrm{E}$. Therefore, it would help in chronic disease treatment caused by oxidative stress. " ' 'kombucha's high antioxidant power prevents body cells damage, inflammatory diseases, immune system failure, and cancerous tumor formation [13, 15, 31]. The chemical structure of some of the polyphenols in kombucha tea is shown in Fig 3 [32].

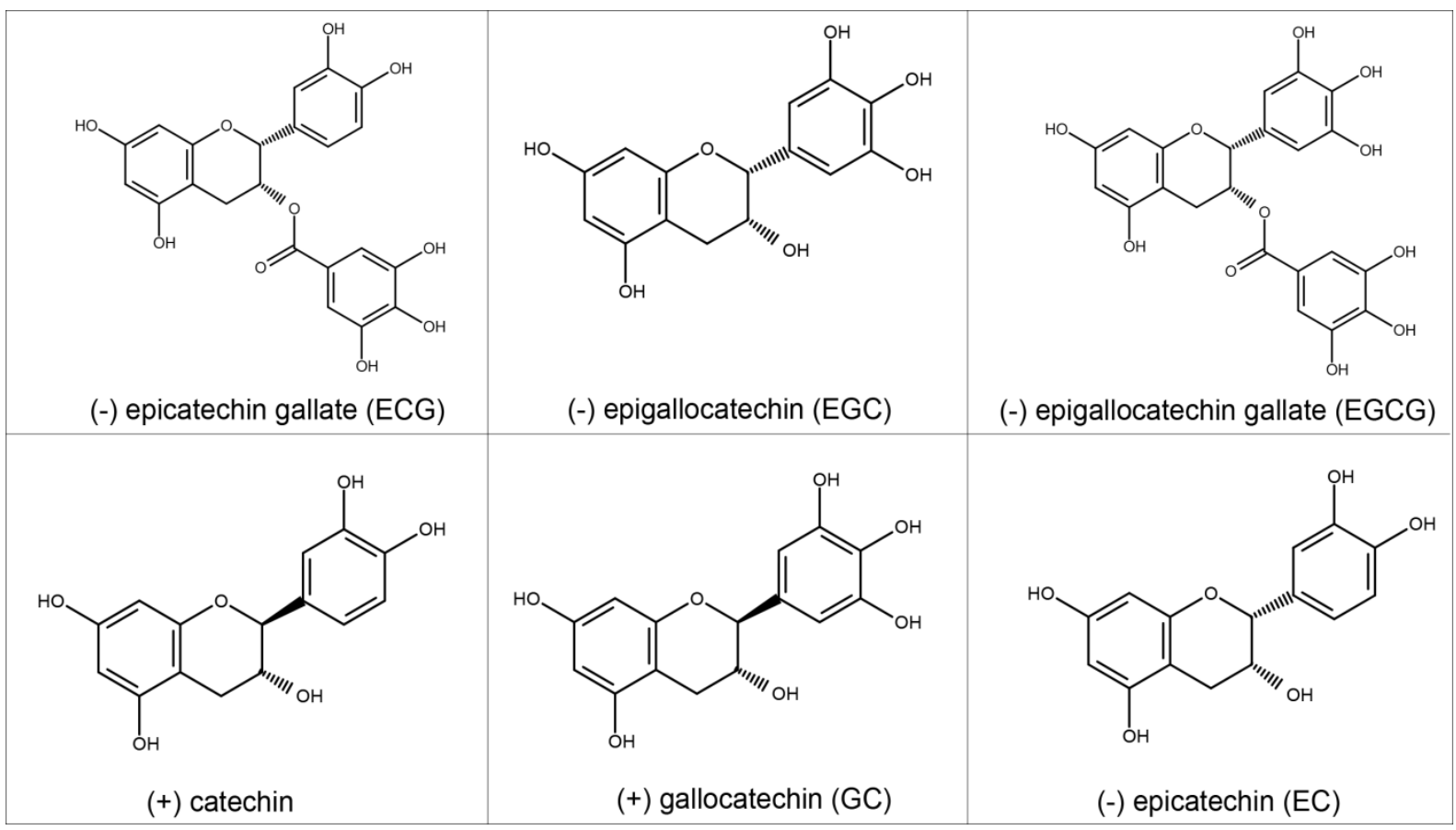

Figure 3. The chemical structure of some polyphenols in Kombucha tea.

\section{Measuring Antioxidant Activity}

\subsection{Cuprac assay.}

The Cuprac assay (Cupric Antioxidant Capacity Reduction) is a new method to measure the total antioxidant capacity (TAC) of hydrophilic and hydrophobic samples. The main reagent, copper (II)-neocoprovin (2,9-dimethyl-10,1-phenoltroline), can oxidize the antioxidants that make the color product. Chelating with neocoprovine allows a faster reaction by increasing the redox potential of the reagent. This method causes oxidation-reduction between the Cupric reagent and antioxidants having a thiol group like glutathione. As shown in Fig 4, in the Cuprac process, the reagent reduces itself and forms a chelate complex of copper (I) -neocoprine, which produces a measurable color at $450 \mathrm{~nm}$ [33]. 


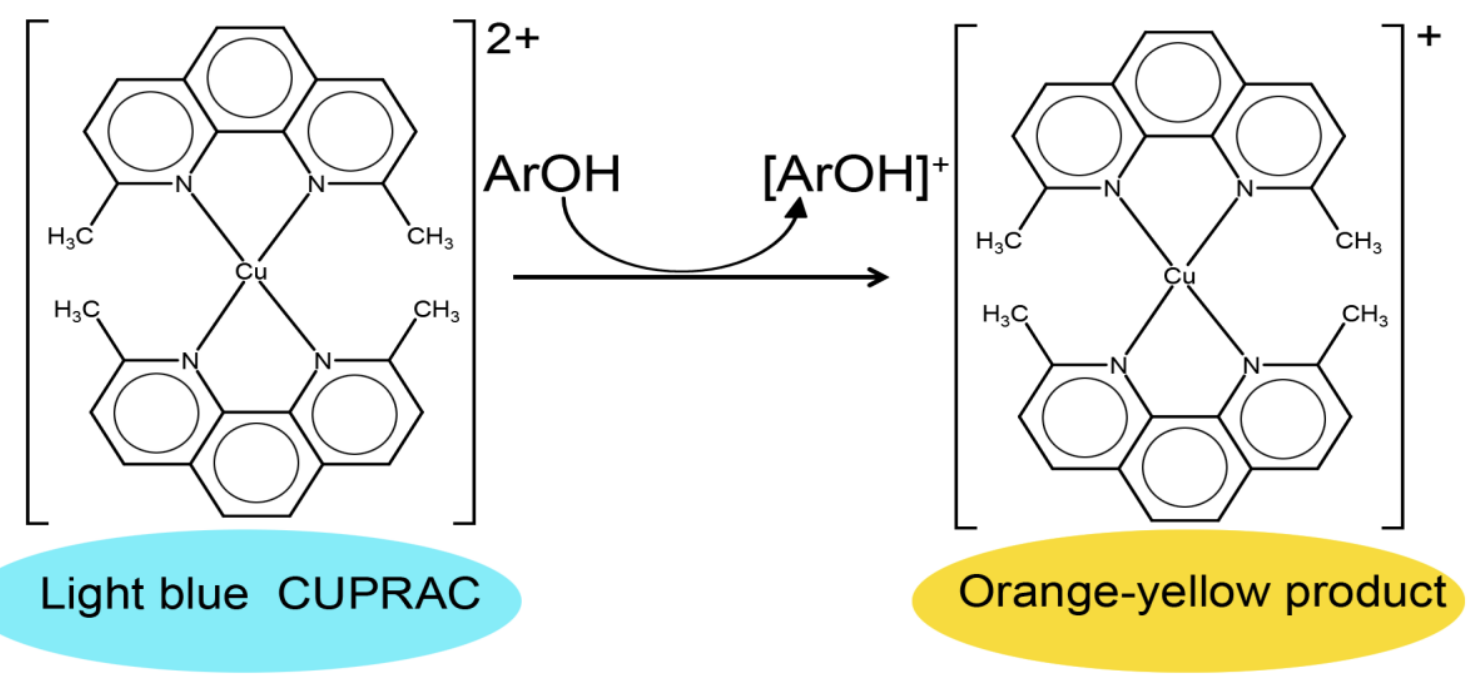

Figure 4. Cuprac reagent reduction reaction.

\subsection{DPPH method.}

Bluis established this method in 1958 as determining the antioxidant activity in a sample using alpha-diphenyl beta-picryl hydrazyl (DPPH) [34]. The method is based on measuring the removal capacity of antioxidants. The electron of nitrogen in DPPH is reduced to the hydrazine by receiving hydrogen atoms from antioxidants (Fig 5) [35].

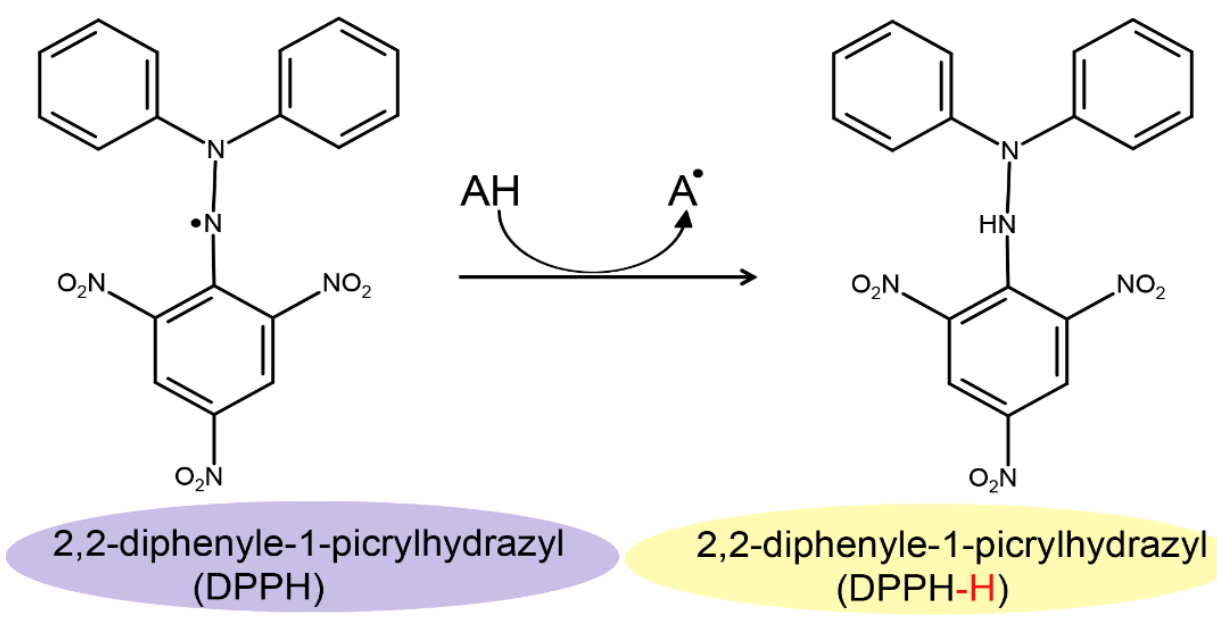

Figure 5. The DPPH radical inhibitory capacity.

\section{Review of Previous Researches}

The published studies of main variables affecting the antioxidant activity and invertase enzyme in kombucha drinks over the last 10 years are given in Table 1.

Bolverdi et al. [36] investigated kombucha drinks with inulin and compared them with the sugar prepared samples. Kombucha with inulin had higher $\mathrm{pH}$, lactic acid content, soluble protein content, and antioxidant activity. Therefore, using inulin as a substrate for kombucha cultivation would shorten the fermentation time, and a drink with higher $\mathrm{pH}$ can be produced.

The ability of kombucha drinks made from green and black tea for inhibiting free radicals was measured. It was revealed that total phenolic compounds, inhibitory activity on DPPH, and hydroxyl radicals increased with increasing the fermentation time, while the lipid peroxidation ability was decreased $[6,37]$. 
Malbasa et al. [38] evaluated two culture starters' effect (combined culture of acetic acid and Zygosaccharomyces, the combined culture of acetic acid and Saccharomyces cerevisiae) on the antioxidant activities of green and black tea kombucha. They assessed their ability to remove hydroxyl radicals and DPPH. The highest antioxidant activity was observed in black and green kombucha tea containing acetic acid along with Zygosaccharomyces.

In another study, the antioxidant and polyphenolic activity of coffee-fortified kombucha was investigated. The solution's $\mathrm{pH}$ was initially 6.8 , and on the first day, it was 4.5, finally, on the fourth day of fermentation, it reached 3.5. Free radical scavenging activity of DPPH increased over time. IC50 in coffee-fortified kombucha ranged from 26.13 to $170.23 \mu \mathrm{l}$ / $\mathrm{ml}[39]$.

Velicanski et al. [9] Measured kombucha's antioxidant and antimicrobial activity with sweetened lemon balm and compared it with traditional kombucha. Rosmarinic acid is the phenolic compound index in this tea. The IC50 level in samples containing sweetened lemon balm was lower than traditional samples and increased from 27.1 on $1^{\text {st }}$ day to 30.2 on day $7^{\text {th }}$ day, and in the traditional kombucha, it increased from 72.3 to 91.2.

In another study, the antioxidant activity and amount of polyphenols in five kombucha tea types during 5 days were determined. Rooibos and Sri Lankan black tea had the highest amount of total phenols before the fermentation process, while black, oolong, and Chinese green tea had the least amount of phenolic compounds. Rooibos, Sri Lankan black tea, and Chinese green tea significantly increased total phenol content from the first day of fermentation. Rooibos and Sri Lankan black tea had the highest total phenol content at the end of the analysis period [40].

In another study, Lobo et al. [41] compared the antioxidant properties and bioactive compounds produced by black and kombucha tea. The results showed higher levels of antioxidants and bioactive compounds in kombucha tea than black tea.

The antioxidant activity was evaluated in kombucha drinks with enhancing the fermentation time. The highest antioxidant activity (93.79\%) was observed on the $7^{\text {th }}$ day of fermentation. The antioxidant activity decreased slightly and reached $93.56 \%$ on the 11th day of fermentation by enhancing the fermentation time. The color of the drink was dark brown at the beginning of fermentation that became lighter over time due to the color decomposition by microorganism's activity. The samples' $\mathrm{pH}$ decreased during fermentation (from 5.93 on the $1^{\text {st }}$ day to 3.65 on the $11^{\text {th }}$ day) [42].

Amarasinghe et al. [5] evaluated the antioxidant activity of four kombucha black tea samples with different concentrations over two months. The $\mathrm{pH}$ of the samples decreased significantly during fermentation, and the samples with higher concentrations of kombucha showed higher antioxidant activities. The antioxidant activity of the samples reduced significantly after the two-month fermentation period. The results revealed that the fermentation period's excessive increase increases the organic acids and makes the drink undesirable.

A comparative study was performed on the antioxidant, microbiology, and biochemistry activity in kombucha tea made from green, black, and rooibos tea. The number of polyphenols and antioxidant activity increased over the fermentation time in all three samples, and both were reached the highest level in green tea on the $7^{\text {th }}$ day [19].

Ahmed et al. [43] studied the biological, chemical, and antioxidant properties of three kombucha types made with black tea, rice, and barley. The free radical scavenging ability of DPPH in tea, barley, and rice kombucha was $86.69 \%, 76.19 \%$, and $36.04 \%$, respectively. The 
total phenolic content of kombucha made from black tea was nearly 3 times more than rice and barely kombucha samples.

Also, Jakubczyk et al. [44] observed the biochemical profile and antioxidant activity in kombucha samples made from green, black, white, and red tea. The fermentation time affected the free radical scavenging properties of kombucha. The analysis of antioxidant potential in samples showed that the amount of antioxidant compounds was between 94.61 to $94.94 \%$ of DPPH radical inhibition.

Recently, a research team has investigated the impact of kombucha incorporation with or without starter culture on yogurt characteristics (including $\mathrm{pH}$ and titratable acidity) during incubation and cold storage $[45,46]$. They showed an increase of the kombucha concentration in all samples, elongated the fermentation period $(\mathrm{p}<0.05)$, Also during the storage, decrease of $\mathrm{pH}$ and increase of acidity were minor $(\mathrm{p}<0.05)$, and with raising the kombucha concentration, these changes were significantly less than control sample $(p<0.05)$.

In a study, the purification and isolation of some microorganisms from kombucha were evaluated by using molecular and phylogenetic methods. The antioxidant activity was measured using DPPH radical inhibition, and it was improved over time [47].

Table 1. The published studies of main variables affecting the antioxidant activity and invertase enzyme in kombucha drink over the last 10 years (2010-2020).

\begin{tabular}{l|l|c} 
Influenced Factors & Media/ Levels & Ref. \\
\hline Carbon source & Sucrose, Honey & {$[48,49]$} \\
\hline Carbon concentration & $5-10 \%$ & {$[41,50]$} \\
\hline Nitrogen source & Black and green tea & {$[41,50]$} \\
\hline Nitrogen concentration & $5-8 \mathrm{gr} / \mathrm{L}$ & {$[49,50]$} \\
\hline Temperature & $24-29^{\circ} \mathrm{C}$ & {$[14]$} \\
\hline pH & $3-5$ & {$[38]$} \\
\hline Fermentation time & $7-14$ days & {$[41,50]$} \\
\hline Antioxidant inducers & Banana peel, & {$[39,51,52]$} \\
\hline Invertase inductor & Cherry juice, Coffee & \\
\hline Starter culture & Orange peel-yeast & {$[53]$} \\
\hline Inoculation rate & Acetic acid bacteria and Saccharomyces cerevisiae & {$[38]$} \\
\hline
\end{tabular}

In this regard, a most recent paper conducted by Jafari et al. [47] conducted the most comprehensive research about all possible process variables on antioxidant and invertase properties of kombucha. They reported the main variables for antioxidant activity were temperature $\left(29^{\circ} \mathrm{C}\right)$, sucrose concentration, inoculation rate, orange peel as invertase inducer, fermentation time 14 days, the most effective factors on increasing antioxidant activity in solution.

\section{Conclusions}

Given the changes in human lifestyle "' 'today's world and the increase in diseases caused by oxidative stress, the simplest and most effective way to overcome this problem is consuming foods with high antioxidant properties in the daily diet. Kambucha is a useful fermented beverage rich in antioxidants such as polyphenols and flavonoids. Sensory acceptance and antioxidant capacity have been the main goal of different studies in kombucha beverages; among them, the majority has been oriented on its antioxidant properties. Also, as the type of microbial population, as well as their viability in the beverage, affects the bioavailability of chemical compounds, the sensory properties, and the overall acceptance, determination of its microbial population has been one of the important aims of many types of 
research. Yeast and acetic acid bacteria are major organisms found in kombucha. Among all the reported yeasts, Saccharomyces cerevisiae is the most promising species to meet the fermentation market's needs due to the lower levels of ethanol, biogenic amines, and volatile acid production. Besides, as enzymes including invertase, amylase, and oxidative enzymes are produced by microorganisms present in kombucha, induction of these enzymatic activities makes this beverage more suitable for specific consumers such as diabetic patients.

Further research on kombucha's sensory properties would lead to a better production process and, therefore, quality. Many studies have also observed and reported the effect of incorporating additives like juice to increase the antioxidant potentiality of this amazing fermented beverage. The literature review shows that some process variables influence the antioxidant properties of kombucha. The $\mathrm{pH}$ of kombucha decreased during fermentation, and meanwhile, the antioxidant activity increased. Also, Invertase activity was enhanced during fermentation, and the antioxidant activity increased with enhancing the invertase activity in kombucha tea. This would make this value more useful for diabetic patients as the antioxidant properties would improve during fermentation.

Further research on the sensory properties of kombucha would lead to a better production process and subsequent quality. More studies are needed to investigate bacterial and yeast species' populations in different tea samples using flow cytometry or specific markers. Also, more research is needed to develop Kombucha beverages in -free forms for those forbidden to consume them due to religious or medical reasons.

\section{Funding}

This research received fund number 27323 from National Nutrition and Food Technology Research Institute, Tehran, Iran.

\section{Acknowledgments}

This research has no acknowledgments.

\section{Conflicts of Interest}

The authors declare no conflict of interest.

\section{References}

1. Shahbazi, H.; Hashemi, H.; Gahruie, M.T.; Golmakani, M.; Eskandari, H.M.; Movahedi, M. Effect of medicinal plant type and concentration on physicochemical, antioxidant, antimicrobial, and sensorial properties of kombucha. Food Sci Nut 2018, 6, 18-22, http://doi.org/10.1002/fsn3.873.

2. Ivanišová, E.; Meňhartová, K.; Terentjeva, M. The evaluation of chemical, antioxidant, antimicrobial and sensory properties of kombucha tea beverage. J Food Sci Technol 2020, 57, 1840-1846, https://doi.org/10.1007/s13197-019-04217-3.

3. Battikh, H.; Chaieb, K.; Bakhrouf, A.; Ammar, E. Antibacterial and antifungal activities of black and green kombucha teas. J Food Biochem 2013, 37, 231-236, https://doi.org/10.1111/j.1745-4514.2011.00629.x.

4. Musial, C.; Kuban-Jankowska, A.; Gorska-Ponikowska, M. Beneficial Properties of Green Tea Catechins. Int. J. Mol. Sci. 2020, 21, https://doi.org/10.3390/ijms21051744.

5. Bhattacharya, D.; Sinha, S.; Mukherjee, P.; Howla, D.R.; Nag, D.; Sarkar, S. Anti-virulence activity of polyphenolic fraction isolated from Kombucha against Vibrio cholera. Microb Pathogen 2020, 140, 11-20, https://doi.org/10.1016/j.micpath.2019.103927.

6. Yan, Z.; Zhong, Y.; Duan, Y.; Chen, Q.; Li, F. Antioxidant mechanism of tea polyphenols and its impact on health benefits. Animal Nut 2020, 6, 115-123, https://doi.org/10.1016/j.aninu.2020.01.001.

7. Laureys, D.; Britton, S.J.; De Clippeleer, J. Kombucha Tea Fermentation: A Review. J Am Soc Brew Chem 2020, 78, 165-174, https://doi.org/10.1080/03610470.2020.1734150. 
8. Villarreal-Soto, S.A.; Beaufort, S.; Bouajila, J.; Souchard, J.P.; Taillandier, P. Understanding the Kombucha Tea Fermentation: A Review. J Food Sci 2019, 83, 508-588, https://doi.org/10.1111/1750-3841.14068.

9. Macedo, R.; De Aryelle, D.C.; De Almeida, L.; Do Amaral, R.Q.; Da Mota, R.N.; De Sousa, P.H.M. Kombucha: Review. Int J Gastro Food Sci 2020, 22, https://doi.org/10.1016/j.ijgfs.2020.100272.

10. Chakravorty, S.; Bhattachary, S.; Bhattachary, S.; Sarkar, S.; Gachhui, R. Kombucha: A Promising Functional Beverage Prepared From Tea. Non-Alco Bev 2019, 6, 285-32, https://doi.org/10.1016/B978-012-815270-6.00010-4.

11. Emiljanowicz, K.E.; Malinowska-Pańczyk, E. Kombucha from alternative raw materials - The review. Crit Rev Food Sci Nutr 2019, 60, 3185-3194, https://doi.org/10.1080/10408398.2019.1679714.

12. Ledormand, P.; Desmasures, N.; Dalmasso, M. Phage community involvement in fermented beverages, an open door to technological advances? Crit Rev Food Sci Nutr 2020, https://doi.org/10.1080/10408398.2020.1790497.

13. Kapp, J.M.; Sumner, W. Kombucha: a systematic review of the empirical evidence of human health benefit. Ann Epidem 2019, 30, 66-70, https://doi.org/10.1016/j.annepidem.2018.11.001.

14. Jayabalan, R.; Malbasa, R.V.; Loncar, E.S.; Vitas, J.S.; Sathishkumar, M. A review on kombucha teamicrobiology, composition, fermentation, beneficial effects, toxicity, and tea fungus. Compr. Rev. Food Sci. Food Saf. 2014, 13, 538-550, https://doi.org/10.1111/1541-4337.12073.

15. Malbasa, R.; Vitas, J.; Loncar, E.; Grahovac, J.; Milanovic, S. Optimisation of the antioxidant activity of kombucha fermented milk products. Czech J Food Sci 2014, 32, 477-484, https://doi.org/10.17221/447/2013-CJFS.

16. Loncar, E.; Djuric, M.; Malbasa, R.; Kolarov, L.G.; Klasnja, M. Influence of working conditions upon kombucha conducted fermentation of black tea. Food Biopro Proc 2006, 84, 186-192, https://doi.org/10.1205/fbp.04306.

17. Vazquez-Cabral, B.D.; Rocha-Guzman, N.E.; Gallegos-Infante, J.A.; Gonzalez-Herrera, S.M.; GonzalezLaredo, R.F.; Moreno-Jimenez, M.R.; Cordova-Moreno, I.T.S. Chemical and sensory evaluation of a functional beverage obtained from infusions of oak leaves (Quercus resinosa) inoculated with the kombucha consortium under different processing conditions. Nutra foods $J$ 2014, 13, 169-78, https://doi.org/10.1007/s13749-014-0035-0.

18. Markov, S.L.; Cvetkovic, D.; Velicanski Aleksandra, S. The availability of alactose mediumfor tea fungus culture and Kombucha fermentation. Arch. Biol. Sci. 2012, 64, 1439-1447, https://doi.org/10.2298/ABS1204439M.

19. Gaggia, F.; Baffoni, L.; Galiano, M. Kombucha beverage from green, black and rooibos teas, a comparative study looking at microbiology. chemistry and antioxidant activity. Nutr 2019, 11, 1-22, https://doi.org/10.3390/nu11010001.

20. Choonut, A.; Saejong, M.; Sangkharak, K. The production of ethanol and hydrogen from pineapple peel by Saccharomyces cerevisiae and Enterobacter aerogenes. Energy Procedia 2014, 52, 242-249, https://doi.org/10.1016/j.egypro.2014.07.075.

21. Jayabalan, R.; Malini, K.; Sathishkumar, M.; Swaminathan, K.; Yun, S.E. Biochemical characteristics of tea fungus producted during kombucha fermentation. Food Sci Biotechnol 2010, 19, 843-847, https://doi.org/10.1007/s10068-010-0119-6.

22. Greenwalt, C.J.; Steinkraus, K.; Ledford, R.A. Kombucha, the fermented tea, microbiology, composition, and claimed health effects. J Food Protect 2000, 63, 976-981, https://doi.org/10.4315/0362-028x-63.7.976.

23. Domizio, P.; Liu, Y.; Bisson, L.; Barile, D. Cell wall polysaccharides released during the alcoholic fermentation by Schizosaccharomyces pombe and S. japonicus, Quantification and characterization. Food Microbiol 2017, 61, 136-149, https://doi.org/10.1016/j.fm.2016.08.010.

24. Dakal, T.C.; Solieri, L.; Giudici, P. Adaptive response and tolerance to sugar and salt stress in the food yeast Zygosaccharomyces rouxii. Int J Food Microbiol 2014, 185, 140-157, https://doi.org/10.1016/j.ijfoodmicro.2014.05.015.

25. Battikh, H.; Bakhrouf, A.; Ammar, E. Antimicrobial effect of kombucha analogues. Food Sci Technol 2012, 47, 71-77, https://doi.org/10.1016/j.lwt.2011.12.033.

26. Chen, C.; BY, L. Studies in microbiological quality and survival of Candida albicans in the tea fungi. $J$ Appel Microb 2010, 89, 834-839.

27. Markov, S.; Malbasa, R.; Hauk, M.; Cvetkovic, D. Investigation of tea fungus microbe assotiations the yeast. Acta Periodica Technological, 2001, 32, 133-138.

28. Tran, T.; Grandvalet, C.; Verdier, F.; Martin, A.; Alexandre, H.; Tourdot-Marechal, R. Microbiological and technological parameters impacting the chemical composition and sensory quality of kombucha. Compr Rev Food Safe 2020, 19, 2050-2070, https://doi.org/10.1111/1541-4337.12574.

29. Beigmohammadi, F.; Karbasi, A.; Beigmohammadi, Z. Production of high glucuronic acid level in kombucha beverage under the influence environmental condition. J Food Technol and Nutr 2010, 7, 30-38.

30. Marsh, A.J.; Sullivan, O.; Hill, C.; Ross, R.; Cotter, P.D. Sequence- based analysis of the bacterial and fungal composition of multiple kombucha (tea fungus) samples. Food Microb 2014, 38, 171- 8, https://doi.org/10.1016/j.fm.2013.09.003. 
31. Gamboa-Gomez, C.; Gonzalez-Laredo, R.; Gallegos-Infante, J.; Perez, M.; Martha, R.; Moreno-Jimenez, M.R.; Flores-Rueda, A.G.; Rocha-Guzman, N.E. Antioxidant and angiotensin-converting enzyme inhibitory activity of eucalyptus camaldulensis and litsea glaucescens infusions fermented with kombucha consortium. Food Technol 2016, 54, 367-374, https://dx.doi.org/10.17113\%2Fftb.54.03.16.4622.

32. Zaveri, N.T. Green tea and its polyphenolic catechins, Medicinal uses in cancer and noncancer applications. Life Sci 2016, 78, 2073-2080, https://doi.org/10.1016/j.lfs.2005.12.006.

33. Apak, R.; Guclu, K.; Ozyurek, M.; Çelik, S.E. Mechanism of antioxidant capacity assays and the CUPRAC (cupric ion reducing antioxidant capacity) assay. Microchimical Acta 2008, 160, 413-419, https://doi.org/10.1007/s00604-007-0777-0.

34. Huh, M.K.; Lee, C.; Moon, S.G. Inhibitory effect of DPPH radical scavenging activity and hydroxyl radicals $(\mathrm{OH})$ activity of chelidonium majus var asiaticum. Int $J$ Adv Multidis Res 2016, 3, 15-22.

35. Kedare, S.B., and Singh, R.P. Genesis and development of DPPH method of antioxidant assay. J Food Sci Technol 2011, 48, 412-422, https://dx.doi.org/10.1007\%2Fs13197-011-0251-1.

36. Bolverdi, M.; Safari, M.; Habibi Rezaee, M.; Hoseini, M. Rezaee, K.; Musavi movahedi, A.A. Production of kombucha by inulin of the gland of jerusalem artichoke. Agriculture Faculty of Tehran University. Sci Tech Mag 2012, 8, 89-100.

37. Cardoso, R.R.; Neto, R.O.; Santos D'Almeida, C.T.; Nascimento, T.P.; Pressete, C.G.; Azevedo, L.; DuarteMartino, H.S.; Cameron, L.C.; Ferreira, M.S.L.; Ribeiro deBarros, F.A. Kombuchas from green and black teas have different phenolic profile, which impacts their antioxidant capacities, antibacterial and antiproliferative activities. Food Res Int 2020, 128, 10-17, https://doi.org/10.1016/j.foodres.2019.108782.

38. Malbasa, R.V.; Loncar, E.S.; Vitas, J.S.; Canadanovic-Brunet, J.M. Influence of starter cultures on the antioxidant activity of kombucha beverage. Food Chem 2011, 127, 1727-1731, https://doi.org/10.1016/j.foodchem.2011.02.048.

39. Essawet, N.A.; Cvetkovic, D.; Velicanski, A. Polyphenol and antioxidant activittes of kombucha beverage enriched with coffeberry extract. Chem Indus Chem Engine 2015, 21, 399-409, https://doi.org/10.2298/CICEQ140528042E.

40. Hoon, L.Y.; Choo, C.; Watawana, M.; Jayawardena, N.; Waisundara, V.Y. Kombucha 'tea fungus' enhances the tea polyphenol contents, antioxidant activity and alphaamylase inhibitory activity of five commonly consumed teas. J Func Foods 2014, 66, 21-28, https://doi.org/10.1016/j.jff.2014.07.010.

41. Lobo, R.O.; Dias, F.; Shenoy, C.K. Kombucha for healthy living, evaluation of antioxidant potential and bioactive compounds. Int Food Res J 2017, 24, 541-546.

42. Nurikasari, M.; Puspitasari, Y.; Siwi, R.P.Y. Characterization and Analysis Kombucha Tea Antioxidant Activity Based On Long Fermentation as a Beverage Functional. J Glob Res Pub Health 2017, 2, 90-96.

43. Ahmed, R.F.; Hikal, M.S.; Abou-Taleb, K.A. Biological, chemical and antioxidant activities of different types kombucha. Ann. Agric. Sci. 2020, 65, 35-41, https://doi.org/10.1016/j.aoas.2020.04.001.

44. Jakubczyk, K.; Kałduńska, J.; Kochman, J.; Janda, K. Chemical profile and antioxidant activity of the kombucha beverage derived from white, green, black and red tea. Antioxidants 2020, 9, 447-462, https://dx.doi.org/10.3390\%2Fantiox9050447.

45. Macvandi, M.; Fadaeii, V.; Khosravi-Darani, K. Effect of Kombucha on pH and titratable acidity of yogurt with or without yogurt starter culture during incubation and cold storage. Nutr. Food Sci 2020, In press.

46. Macvandi, M.; Fadaeii, V.; Khosravi-Darani, K. Incorporation of komucha with or without yogurt starter culture during fermentation. Food Sci Technol 2020, In press.

47. Jafari, R.; Naghavi, N.; Khosravi-Darani, K.; Doudi, M.; Shahanipour, K. Kombucha microbial starter with enhanced production of antioxidant compounds and invertase. Biocat Agr Biotech 2020, 29, 101-107, https://doi.org/10.1016/j.bcab.2020.101789.

48. Naffe-Skocinska, K.; Sionek, B. Scibisz, L.; Kolozyn-Krajewska, C. Acid content and the effect of fermentation condition of kombucha tea beverage on physiochemical, microbiological and sensory properties. CYTA J food 2017, 15 601-607, https://doi.org/10.1080/19476337.2017.1321588.

49. Caili, F.U.; Yan, F.; Cao, Z.; Xie, F.; Lin, J. Antioxidant activities of kombucha prepared from three different substrates and changes in content of probiotics during storage. Food Sci Technol 2014, 34, 123-126, https://doi.org/10.1590/S0101-20612014005000012.

50. Goh, W.N.; Rosma, A.; Kaur, A.; Fazilah, A.; Karim, A.; Rajeev, B. Fermentation of black tea broth (kombucha), I. effects of sucrose concentration and fermentation time on the yield of microbial cellulose. Int Food Res J 2012, 19, 109-117.

51. Ebrahimi Pure, A.; Ebrahimi Pure, M. Antioxidant and antibacterial activity of kombucha beverages Prepared using banana peel, common nettles and black tea infusions. Appl Food Biotech 2016, 3, 125-130, https://doi.org/10.22037/afb.v3i2.11138.

52. Yavari, N.; Mazaheri Assadi, M.; Larijani, K.; Moghadam, M.B. Response surface methodology for optimization of glucuronic acid production using kombucha layer on sour cherry juice. Aus J Bas Appl Sci 2010, 4, 3250-3256.

53. Shankar, T.; Thangamathi, P.; Rama, R.; Sivakumar, T. Optimization of Invertase Production Using Saccharomyces Cerevisiae MK Under Varying Cultural Conditions. Int J Biochem Biophys 2013, 1, 47-56. 